\title{
Genehmigungsbedürftige Leistungen nach der Vereinbarung zur Strahlendiagnostik und -therapie
}

\section{Einführung \\ $\nabla$}

Vertragsärztlich tätigen Radiologen drohen nach einer bisher in der Praxis wenig beachteten Entscheidung des Sozialgerichtes Marburg vom 20.07.2011 (Az.: S 12 KA 286/10) Honorarverluste, wenn sie bei der erstmaligen Installation der technischen Ausstattung oder bei der Gründung von Zweigpraxen (Nebenbetriebsstätten) keine Genehmigung zur Durchführung und Abrechnung von radiologischen Leistungen nach den seitens der Partner der Bundesmantelverträge gemäß § 135 Abs. 2 SGB V vereinbarten Qualitätssicherungsvereinbarungen besitzen.

Dies gilt nach der Vereinbarung zur Strahlendiagnostik und -therapie auch dann, wenn bei Veränderungen der technischen Ausstattung nicht rechtzeitig eine Änderung der Genehmigung zur Durchführung und Abrechnung von Leistungen beantragt wurde. Besonders im Fachgebiet der Radiologie bedarf ein Vertragsarzt neben seiner Zulassung zur vertragsärztlichen Versorgung einer Reihe von Genehmigungen, um bestimmte vertragsärztliche Leistungen erbringen und abrechnen $\mathrm{zu}$ dürfen. So bestehen neben der Vereinbarung zur Strahlendiagnostik und -therapie gemäß § 135 Abs. 2 SGB V weitere Qualitätssicherungsvereinbarungen wie die Qualitätssicherungsvereinbarung zur interventionellen Radiologie, die Kernspintomografie-Vereinbarung, die Qualitätssicherungsvereinbarung zur MR-Angiografie, die Ultraschall-Vereinbarung, die Mammografie-Vereinbarung sowie nach $\S 75$ Abs. 7 SGB V weitere Richtlinien der Kassenärztlichen Bundesvereinigung für Verfahren zur Qualitätssicherung. Der vertragsärztlich zugelassene oder ermächtigte Radiologe sowie ein Medizinisches Versorgungszentrum (MVZ) haben ohne eine entsprechende Genehmigung keinen Vergütungsanspruch gegenüber der Kassenärztlichen Vereinigung für die nicht oder nicht mehr genehmigten radiologischen Leistungen (Anmerkung: In der RöFo 2012, Seiten 848 bis 851 haben wir schwerpunktmäßig die Voraussetzungen für die Erteilung und den
Widerruf von Abrechnungsgenehmigungen in der Radiologie nach $\S 135$ Abs. 2 SGB V behandelt. Der Schwerpunkt jenes Beitrages lag in dem Widerruf, den Widerrufsfolgen und den Rechtsmitteln gegen einen Widerruf. Dieser Beitrag setzt sich mit dem Erfordernis der Genehmigung und Veränderungen in der Ausübung der radiologischen Tätigkeit hinsichtlich der apparativen Ausstattung und räumlicher Veränderungen auseinander).

\section{Sachverhalt der Entscheidung $\nabla$}

Der Kläger war als Facharzt für Radiologie zur vertragsärztlichen Versorgung zugelassen. Er besaß eine Genehmigung zur Durchführung und Abrechnung von computertomografischen Leistungen von der zuständigen Kassenärztlichen Vereinigung. In der Genehmigung waren die apparative Ausstattung, ein Computertomograf Typ A und der Standort des Gerätes angegeben. Anfang Mai 2009 teilte der Kläger der zuständigen Kassenärztlichen Vereinigung unter Vorlage der entsprechenden Anzeigenbestätigung des Regierungspräsidiums der Stadt, als der zuständigen Behörde nach § 3 Abs. 1 Röntgenverordnung mit, dass ein Wechsel der apparativen Ausstattung zur Monatsmitte vorgenommen werden solle. Der Wechsel sollte nicht von dem Computertomograf Typ A, sondern vom Typ B zu Typ C erfolgen. $\mathrm{Er}$ beantragte eine entsprechende Genehmigung rückwirkend zum 01.03.2009. Bereits im Jahre 2007 war der Computertomograf Typ B der zuständigen Kassenärztlichen Vereinigung durch einen anderen Arzt der Gemeinschaftspraxis mitgeteilt worden, als die Praxis noch nicht als Gemeinschaftspraxis, sondern lediglich als Apparategemeinschaft betrieben worden war. Der Arzt hatte erklärte, der Computertomograf Typ B werde in der Apparategemeinschaft betrieben, in der der spätere Kläger Gesellschafter war. Die Kassenärztliche Vereinigung erteilte dem Kläger eine Genehmigung zur Durchführung und Abrechnung computertomografischer Leistungen mit der apparativen Ausstattung Typ B, obwohl dieser die Ge-

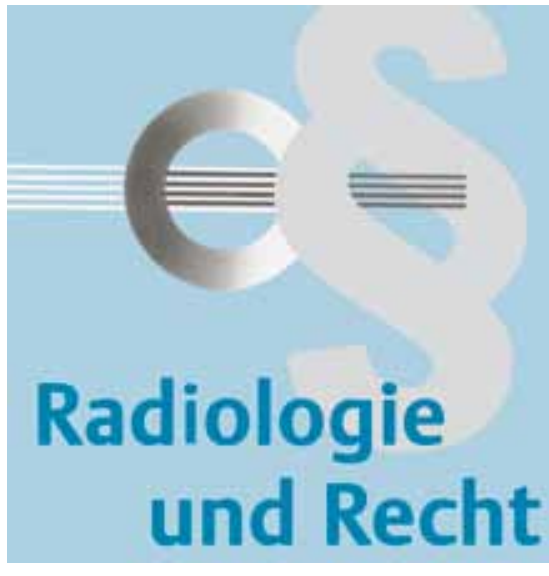

nehmigung des Typ C beantragt hatte. Gegen die Genehmigung erhob der Kläger zunächst Widerspruch und später - erfolglos - Klage vor dem Sozialgericht Marburg.

\section{Inhalt der Entscheidung}

Nach der Vereinbarung zur Strahlendiagnostik und -therapie ist die Ausführung und Abrechnung von Leistungen der diagnostischen Radiologie, der Strahlentherapie und Nuklearmedizin im Rahmen der vertragsärztlichen Versorgung durch die an der vertragsärztlichen Versorgung teilnehmenden Ärzte erst nach Erteilung der Genehmigung durch die Kassenärztliche Vereinigung zulässig. § 2 der Vereinbarung zur Strahlendiagnostik und - therapie über die „Genehmigungspflicht“ lautet:

„Die Ausführung und Abrechnung von Leistungen der diagnostischen Radiologie, der Strahlentherapie und Nuklearmedizin im Rahmen der vertragsärztlichen Versorgung durch die an der vertragsärztlichen Versorgung teilnehmenden Ärzte ist erst nach Erteilung der Genehmigung durch die Kassenärztliche Vereinigung zulässig. [...]“

Die Genehmigung ist zu erteilen, wenn der Arzt die Erfüllung der Voraussetzungen der fachlichen Befähigung (Abschnitt B der Vereinbarung) und der apparativen Ausstattung (Abschnitt $\mathrm{C}$ und Anlagen I bis III der Vereinbarung) nachweist. Das Verfahren richtet sich nach Abschnitt D der Vereinbarung zur Strahlendiagnostik und -therapie ( $§ 3$ Satz 1 und 2). Auf die Details des Verfahrens nach der Vereinbarung kam es in der Entscheidung des Sozialgerichtes Marburg nicht an. 
Dem Antrag auf Genehmigung zur Ausführung und Abrechnung von Leistungen der diagnostischen Radiologie sind nach $\S$ 14 Abs. 2 Satz 1 Nr. 1 bis 4 der Vereinbarung zur Strahlendiagnostik und -therapie insbesondere u.a. beizufügen:

- Zeugnisse gemäß § 16 Abs. 1 oder 2 für den Nachweis der fachlichen Qualifikation; erforderliche Bescheinigungen für den Strahlenschutz;

- Nachweis der Erfüllung der Anforderungen an die apparative Ausstattung gemäß den Anlagen I bis III, der Nachweis kann durch die Gewährleistung des Herstellers, dass das verwendete Gerät diesen Anforderungen entspricht, geführt werden;

- die Genehmigung nach $\S 3$ Abs. 1 der Röntgenverordnung oder die Betriebserlaubnis (Bauartzulassung des Röntgenstrahlers und Strahlenschutzmaßnahmen) nach $\S 4$ Abs. 1 der Röntgenverordnung.

Zum Nachweis der Erfüllung der Anforderungen an die apparative Ausstattung gehört auch die Anzeigenbestätigung der Aufsichtsbehörde nach $\S 3$ Abs. 1 der Röntgenverordnung. Diese Unterlagen legte der Radiologe der Kassenärztlichen Vereinigung erst am 11.05.2009 vor.

Dem Kläger war nur die Durchführungsund Abrechnungsgenehmigung für den Computertomograf Typ A mit Bescheid vom 10.12.2007 erteilt worden. Der Kläger argumentierte gegenüber dem Sozialgericht Marburg, dass ein anderer Gesellschafter der Apparategemeinschaft, die der Kassenärztlichen Vereinigung zuvor angezeigt worden war, einen Genehmigungsantrag für den Computertomograf Typ B gestellt habe. Er meinte, der Antrag des Mitgesellschafters der Apparategemeinschaft gelte zugleich für ihn, weil die Kassenärztliche Vereinigung von der Apparategemeinschaft Kenntnis hatte. Das Sozialgericht Marburg folgte dieser Argumentation nicht, sondern war der Auffassung, dass von der Kassenärztlichen Vereinigung als Träger öffentlicher Verwaltung nicht verlangt werden könne, dass sie aufgrund des Hinweises, das Gerät werde in einer Apparategemeinschaft betrieben, erkennen müsse, dass auch für den in der Apparategemeinschaft tätigen Arzt ein Antrag auf Genehmigung gestellt werden solle. Die Kassenärztliche Vereinigung hatte letztlich erst durch ein Schreiben des klagenden Radiologen am 07.09.2009 erfahren, dass dieser einen bis dahin für seine Person vertragsärztlich ungenehmigten Computertomograf einsetzte.

Für das Sozialgericht Marburg war ein weiterer Aspekt von Bedeutung, der unabhängig von den Fragen der Apparategemeinschaft bestand. Entscheidend sei, dass die Unterlagen für die Genehmigung erst mit der Vorlage der Anzeigebestätigung der Behörde nach $\S 3$ Abs. 1 Röntgenverordnung am 11.05.2009 vollständig bei der Kassenärztlichen Vereinigung vorlagen. Nach den Vorschriften der Vereinbarung zur Strahlendiagnostik und -therapie seien die Unterlagen vor Erteilung der Genehmigung zu überprüfen und die Ausführung und Abrechnung von Leistungen der Leistungen der diagnostischen Radiologie erst nach Erteilung der Genehmigung durch die Kassenärztliche Vereinigung zulässig. Eine Erteilung der Genehmigung vor einer Vorlage der erforderlichen Unterlagen könne nicht erfolgen. Wie in der Praxis häufig, erfolgte die Genehmigung nicht lediglich für die Zukunft nach Wirksamwerden des Durchführungs- und Abrechnungsbescheides, sondern rückwirkend ab dem Zeitpunkt des Eingangs des letzten geforderten Nachweises. Eine darüber hinausgehende Möglichkeit, die Genehmigung für die Vergangenheit zu erteilen, besteht nach der Vereinbarung zur Strahlendiagnostik und -therapie nicht. Dies gilt nach Auffassung des Sozialgerichts Marburg generell für die Genehmigungserteilung nach Vereinbarungen zur Qualitätssicherung, soweit die Genehmigung wie im Falle der Vereinbarung zur Strahlendiagnostik und -therapie als Maßnahme der Qualitätssicherung ausdrücklich als Abrechnungsvoraussetzung formuliert sei.

Radiologisch tätige Vertragsärzte sollten unbedingt beachten, dass im System der vertragsärztlichen Leistungserbringung der Grundsatz gilt, dass eine für bestimmte spezialisierte Leistungen erforderliche Genehmigung vor der Leistungserbringung erteilt sein muss und weder rückwirkend erteilt werden, noch nach ihrer Erteilung Rückwirkungen für einen vor der Erteilung liegenden Zeitpunkt entfalten kann (vgl. Bundessozialgericht, Urteil vom 28.01.1998, Az.: B 6 KA 93/96 R; Urteil vom 28.01.1998, Az.: B 6 KA 41/96 R). Die Unzulässigkeit einer Rückwirkung wird in der Rechtsprechung des Bundessozialgerichtes damit begründet, dass sich dies aus dem System des Vertragsarztrechts, das nach wie vor durch das Naturalleistungsprinzip in Verbindung mit der
Beschränkung der Leistungserbringung auf einen umgrenzten Kreis dafür qualifizierter Leistungserbringer geprägt sei, ergebe. Mit dieser Beschränkung sei verbunden, dass den Vertragsärzten die Berechtigung zur Erbringung von Leistungen förmlich zuerkannt worden sein müsse (vgl. Bundessozialgericht, Urteil vom 28.01.1998, a.a.O.).

\section{Genehmigung einer Zweigpraxis $\nabla$}

Im Zusammenhang mit Zweigpraxen besteht in der Praxis häufig ein vermeidbarer Irrtum, der auf die Verteilung der Zuständigkeiten zwischen dem Zulassungsausschuss für Ärzte und der Kassenärztlichen Vereinigung zurückgeht. Soll z.B. durch einen angestellten Arzt, der bei einem vertragsärztlich tätigen Radiologen tätig ist, in einer Zweigpraxis Sprechstunden abgehalten werden, bedarf es für diese Tätigkeit ebenfalls der Genehmigung der Kassenärztlichen Vereinigung nach $\S$ 24 Abs. 3 Ärzte-ZV. Sofern die Zweigpraxis außerhalb des Bezirks der Kassenärztlichen Vereinigung liegt, ist für die Erteilung der Genehmigung der Zulassungsausschuss zuständig, in dessen Bezirk der angestellte Arzt die Tätigkeit aufnehmen will. Sollen darüber hinaus Leistungen in der Zweigpraxis erbracht werden, für die eine Qualitätssicherungsvereinbarung nach § 135 Abs. 2 SGB V existiert, wie durchgängig im radiologischen Bereich, bedarf es zusätzlich einer Abrechnungsgenehmigung für die Leistungen durch die Kassenärztliche Vereinigung, in dessen Zuständigkeitsbereich die Zweigpraxis liegt.

Häufiger ist allerdings der Fall anzutreffen, dass ein Vertragsarzt aufgrund einer durch die Kassenärztliche Vereinigung erteilte Durchführungs- und Abrechnungsgenehmigung für eine Zweigpraxis in dem Glauben ist, dass damit auch die Tätigkeit in der Zweigpraxis genehmigt wäre. Die Kassenärztliche Vereinigung prüft durch die zuständige Abteilung der Qualitätssicherung nur, ob die 3 Kriterien der Qualitätssicherungsvereinbarungen erfüllt werden: besondere Kenntnisse und Erfahrungen, eine besondere Praxisausstattung und Anforderungen an die Versorgungsqualität. Ob die Voraussetzungen nach der Ärzte-Zulassungsverordnung vorliegen, muss dagegen ungeachtet der Qualität der Zweigpraxis die Sicherungsstellungsabteilung bzw. der Zulassungsausschuss feststellen. Vor Beginn der Durchführung 
und Abrechnung von Leistungen in einer Zweigpraxis, die der Genehmigung einer Qualitätssicherungsvereinbarung unterliegen, müssen daher 2 Genehmigungsbescheide vorliegen.

\section{Zusammenfassung und Ausblick $\nabla$}

Die besonderen Genehmigungsanforderungen für Leistungen nach den Qualitätssicherungsvereinbarungen gemäß $§ 135$ Abs. 2 SGB V bestehen nach der Rechtsprechung des Bundessozialgerichtes für den gesamten Bereich der Durchführungsund Abrechnungsgenehmigungen. Vor der Durchführung und Abrechnung von Leistungen, die nach einer Qualitätssicherungsvereinbarung genehmigungspflichtig sind, muss daher die Antragstellung auf Erteilung der Genehmigung unter Vorlage sämtlicher geforderter Unterlagen erfolgen. Sie mag in vielen Bereichen ein überzogener Formalismus sein, weil die betroffenen Belange seitens der Kassenärztlichen Vereinigungen nicht unmittelbar erkennbar sind und Risiken für die Versicherten nicht bestehen, weil die Genehmigung für den Betrieb von Röntgeneinrichtungen nach $\S 3$ Abs. 1 RöV durch die zuständige Behörde bereits vorher erteilt worden sein muss. Der Einsatz eines durch die Kassenärztliche Vereinigung nicht genehmigten Computertomograf oder Kernspintomograf steht der Abrechnung von Leistungen, die mit diesem Gerät erbracht wurden, an gesetzlichen krankenversicherten Patienten daher entgegen, sodass der Vergütungsanspruch seit der Inbetriebnahme vollständig, ggf. bei späterer Kenntnis der Kassenärztlichen Vereinigung auch rückwirkend entfällt. Daher sollten Radiologen bei jeder Änderung der apparativen Ausstattung nicht nur an die Anzeige gegenüber der nach $\S 3$ Abs. 1 RöV zuständigen Behörde, sondern in der vertragsärztlichen Versorgung auch an die Neuerteilung der Durchführungs- und Abrechnungsgenehmigung nach der jeweiligen Qualitätssicherungsvereinbarung gemäß $\S 135$ Abs. 2 SGB V denken. Soweit Vertragsärzte im
Rahmen von Zweigpraxen tätig werden, ist unbedingt darauf zu achten, dass sowohl der Genehmigungsantrag für die Tätigkeit in der Zweigpraxis nach $§ 24$ Abs. 3 Ärzte-ZV, als auch der Antrag auf Erteilung bzw. Ergänzung einer Durchführungs- und Abrechnungsgenehmigung durch die Abteilung Qualitätssicherung der jeweiligen Kassenärztlichen Vereinigung erteilt wird.

Das Sozialgericht Marburg hat in seiner Entscheidung hervorgehoben, dass von der Kassenärztlichen Vereinigung nicht verlangt werden könne, dass sie aufgrund des Hinweises, das Gerät werde in einer Apparategemeinschaft betrieben, hieraus schließen müsse, dass auch für den in der Apparategemeinschaft tätigen Arzt ein Antrag auf Genehmigung gestellt werden soll. Gleiches gilt auch für eine Berufsausübungsgemeinschaft nach $\S 33$ Abs. 2 Ärzte-ZV und ein Medizinisches Versorgungszentrum nach $\S 95$ Abs. 1 SGB V, wenn es zu Änderungen der apparativen Ausstattung kommt. Für jeden in einer BAG oder einem MVZ tätigen Radiologen muss daher eine Abrechnungsgenehmigung bei der Kassenärztlichen Vereinigung eingeholt werden.

René T. Steinhäuser

Rechtsanwalt

Rechtsanwälte Wigge

Neuer Wall 44

20354 Hamburg

Telefon: (040) 3398705-90

Telefax: (040) 3398705-99

Internet: www.ra-wigge.de

E-Mail: kanzlei@ra-wigge.de 